\title{
Influência da radiação gama e de diferentes dietas na qualidade da carne de cordeiros Santa Inês
}

\author{
Influence of the radiation and of different diets on quality of meat lambs Santa Ines
}

Adriana Régia Marques de SOUZA ${ }^{1}$; Valter ARTHUR ${ }^{1}$; Solange Guidolin CANNIATTI-BRAZACA ${ }^{2 *}$

\begin{abstract}
Resumo
O processo de irradiação é um método eficiente de conservação de alimentos, pois reduz o número de microrganismos patogênicos e deteriorantes. Com doses baixas, as características organolépticas e nutricionais do alimento não são alteradas significativamente, principalmente quando são controlados outros fatores como, por exemplo, a embalagem. Os objetivos deste trabalho foram avaliar a cor, capacidade de retenção de água (CRA) e formação de bases voláteis na carne de cordeiros Santa Inês tratados com diferentes dietas e doses de radiação. Amostras de carne de cordeiro tratados com diferentes dietas - controle, TAC1, TAC2 (taninos altamente condensados) e sorgo - foram embaladas a vácuo e, excetuando-se a controle (sem irradiação), irradiadas nas doses 2 e 4 kGy e armazenadas por 15 dias à temperatura de $4{ }^{\circ} \mathrm{C}$. As medidas de $a^{*}$ (redness) e $b^{*}$ (yelowness) não sofreram influência das diferentes dietas e doses empregadas no experimento. Os valores de $L^{*}$ (lightness) se mostraram diferentes para dietas no controle (sem irradiação) e na dose $2 \mathrm{kGy}$, mas, quando comparados os efeitos das doses em cada dieta, estas não apresentaram diferenças. Os valores de $H$ e $C$ calculados não mostraram diferenças estatísticas. O tempo de armazenamento influenciou as medidas de $a^{*}, b^{*}, L, H$ e $C$. A irradiação diminuiu os teores de bases voláteis e CRA, porém o tempo de estocagem aumentou-os.
\end{abstract}

Palavras-chave: irradiação; cordeiro; cor; taninos; voláteis; textura.

\begin{abstract}
The irradiation process is an effective method for food preservation because it reduces the pathogenic and/or the number of deteriorating microorganisms. The use of low doses did not alter the organoleptic and sensorial food characteristics significantly, especially when other factors such as packaging are considered. The objective of this work is to evaluate the color, water holding capacity (WHC), and formation of volatile bases in the meat of Santa Ines lambs treated with different diets and radiation doses. Lamb samples treated with different diets: control, TAC1, TAC2 (tannins highly condensed), and sorghum were vacuum wrapped, irradiated in the doses 2 and $4 \mathrm{kGy}$ and control (without irradiation), and stored under $4^{\circ} \mathrm{C}$ for 15 days. The values of $a^{\star}$ (redness) and $b^{\star}$ (yellowness) were not influenced by the different diets and doses used in the experiment. The values of $L^{\star}$ (lightness) differed statistically for the control diets (without irradiation) and for $2 \mathrm{kGy}$ dose diet. However, the effects of the doses in each diet were not different. The values of $\mathrm{H}$ and $\mathrm{C}$ values obtained did not show statistical differences. The storage time influenced the measures of $a^{*}, b^{*}, L, \mathrm{H}$, and $\mathrm{C}$. The irradiation reduced the levels of the volatile bases and water holding capacity (WHC) although these parameters were increased due to the storage time.

Keywords: irradiation; sheep; color; tannin; volatile; texture.
\end{abstract}

\section{Introdução}

A irradiação pode eliminar os microrganismos patogênicos da carne e tornar os alimentos seguros para o consumo, além de aumentar seu tempo de estocagem devido à diminuição da flora bacteriana. Estudos têm relatado os efeitos da irradiação na qualidade da carne e também mudanças na coloração relacionadas com a dose, tipo de carne e embalagem (LIU et al., 2003). Os efeitos da radiação ionizante dependem da dose total absorvida, taxa de absorção e as condições do meio, como a temperatura, presença de oxigênio, entre outras, no momento da irradiação (BREWER, 2004).

A identificação dos pigmentos gerados pela irradiação ainda não são claros. Estudos mostram que a coloração vermelha em carne fresca de porco e peru é devida à formação de oximioglobina. A coloração vermelha, todavia, pode ser produzida por várias condições. A embalagem é um fator muito importante na coloração, permitindo ou não a formação da coloração vermelha (NAM; AHN, 2002).

A coloração da carne é devida à concentração de pigmentos (mioglobina e hemoglobina) e o estado químico desses pigmentos (LAWRIE, 2002). Mioglobina é uma metaloproteína composta de globina e ferro contendo o grupo prostético heme. A globina pode estar no seu estado natural ou desnaturado, o átomo de ferro pode estar em vários estados de oxidação e a porfirina pode estar com seu anel intacto, oxidado, polimerizado

Recebido para publicação em 13/9/2007

Aceito para publicação em 3/1/2009 (002834)

${ }^{1}$ Laboratório de Irradiação de Alimentos e Radioentomologia, Centro de Energia Nuclear na Agricultura, CENA/USP, E-mail: acornel@cena.usp.bre arthur@cena.usp.br

${ }^{2}$ Laboratório de Nutrição Humana, Departamento de Agroindústria, Alimentos e Nutrição, Escola Superior de Agricultura Luiz de Queiroz, ESALQ/USP,

E-mail: sgcbraza@esalq.usp.br

${ }^{*}$ A quem a correspondência deve ser enviada 
ou aberto. A mioglobina está relacionada com a função biológica: estocar oxigênio necessário para os tecidos e aumentar a taxa de difusão de oxigênio pela célula quando for necessário o aumento da eficiência para a contração muscular (ANDERSON, 2005). $\mathrm{O}$ oxigênio estocado permite ao grupo heme a habilidade para sofrer reações de oxirredução e transferência de elétrons (BREWER, 2004).

O maior interesse na irradiação de carnes, entretanto, é o efeito na qualidade da carne, principalmente porque as reações com radicais livres resultam na possibilidade de geração de odores. A radiação ionizante gera radicais hidroxi em meio aquoso, que podem afetar os lipídios presentes no meio (BREWER, 2004). Estes radicais hidroxi podem ser produzidos na irradiação de carnes e desencadear alteração nos lipídios já que significativa porção de células musculares (75\%) é cercada por lipídios. Os compostos voláteis responsáveis pelos odores produzidos pelas mudanças na proteína e moléculas lipídicas na irradiação são diferentes dos odores produzidos pela oxidação lipídica (AL-BACHIR; MEHIO, 2001).

A presença de antioxidantes aumenta a estabilidade da cor da carne irradiada (XIONG et al., 1993). Alguns antioxidantes fenólicos, tais como a vitamina $\mathrm{E}$, inativam os radicais formados minimizando os danos autoxidativos na carne provocados pelo processo de irradiação. Esses antioxidantes podem ser ministrados aos animais ou também ser adicionados diretamente na carne antes do processo de irradiação (NAM; PRUSA; AHN, 2002; NAM et al., 2003).

Os taninos pertencem ao grupo dos polifenóis e apresentam também capacidade antioxidante. Portanto, a alimentação de cordeiros com dietas ricas em taninos condensados é uma alternativa para o controle de algumas verminoses e também podem atuar como antioxidante na carne dos animais.

Taninos condensados são compostos fenólicos encontrados em várias forrageiras e árvores. Em baixas concentrações, alguns taninos condensados podem conferir vantagens nutricionais para ruminantes por redução de proteínas degradadoras no rúmen e aumento da circulação de proteína e aminoácidos essenciais no intestino. O sorgo, como também o extrato de acácia, contém altos níveis de tanino e outros compostos fenólicos, sua utilização na alimentação dos animais eleva o conteúdo de antioxidantes na carne. Taninos e compostos fenólicos relacionados têm grande efeito antioxidante nos músculos do animal, melhorando a estabilidade oxidativa da carne (DU et al., 2002). Esses compostos sequestram radicais livres, por isso são eficazes para prevenir a oxidação lipídica (SOARES, 2002). Porém, taninos e outros compostos fenólicos podem formar um complexo insolúvel com o ferro no trato intestinal e, com isso, reduzir a biodisponibilidade de ferro solúvel nas carnes dos animais (BRUNE; ROSSANDER; HALLBERG, 1989).

A indústria de carnes se preocupa muito com os consumidores, por isso qualquer aspecto que possa causar desconfiança ou rejeição do produto é de interesse da indústria. Uma medida de interesse é a capacidade de retenção de água (CRA), que é considerada um índice importante de qualidade, pois a quantidade de água existente na carne pode afetar seu valor comercial além de estar relacionada diretamente com as propriedades sensoriais de suculência, textura e sabor da carne (SILVA, 2004).

Tendo em vista a manutenção da qualidade da carne com a utilização da irradiação, os objetivos deste trabalho foram avaliar a cor, a capacidade de retenção de água e a formação de bases voláteis na carne de cordeiros Santa Inês tratados com diferentes dietas e doses de radiação.

\section{Material e métodos}

\subsection{Material}

Cordeiros da raça Santa Inês foram divididos em quatro grupos com 4 animais por grupo, sendo o grupo 1 (controle) tratado com feno e concentrado proteico (50\%-50\%); grupos (2 e 3) tratados com taninos altamente concentrados (extrato de Acácia molissima), que receberam 1,6 g de taninos por quilo de peso vivo (nove dias antes do abate), sendo que o grupo 2 recebeu tanino uma vez (TAC1), e o grupo 3 recebeu duas vezes (TAC2); e o grupo 4 foi tratado com sorgo como fonte de taninos. Todos os grupos receberam água ad libitum. Os animais foram abatidos com 4 meses de idade e foi separada a paleta, a qual foi mantida sob refrigeração por 48 horas. As amostras foram desossadas e separadas em alíquotas ( $\pm 50 \mathrm{~g}$ ) e embaladas a vácuo, em embalagens de polietileno de baixa densidade, levadas então para armazenamento a $4{ }^{\circ} \mathrm{C}$ e irradiadas.

O irradiador utilizado no experimento foi o Multipropósito do Ipen (Instituto de Pesquisas Energéticas e Nucleares), com fonte de ${ }^{60} \mathrm{Co}$ e a taxa de dose foi de $6 \mathrm{kGy} / \mathrm{h}$. As amostras receberam doses de 2 e $4 \mathrm{kGy}$ na faixa de temperatura de 0 a $2{ }^{\circ} \mathrm{C}$. As amostras não irradiadas serviram de controle. Todas as amostras foram refrigeradas a $4{ }^{\circ} \mathrm{C}$ e estocadas por 15 dias.

\subsection{Análise de cor}

A cor da carne foi medida usando-se um aparelho de espectrofotômetro portátil (Colorímetro Minolta) e os valores foram expressos em $L$ (luminosidade), $a^{*}$ (vermelho) e $b^{*}$ (amarelo). Os valores de $C$, que indicam a pureza da cor dentro da escala do cinza a variante mais viva da cor percebida; e de $H$ ou tonalidade quando os valores do ângulo hue estão próximos de $0^{\circ}$, a cor é púrpura, quando estão próximos de $90^{\circ}$ apresenta coloração amarelada, ou verde-azulada $\left(180^{\circ}\right)$ ou azuis $\left(270^{\circ}\right)$. Estas características de coloração foram calculadas usandose as Equações 1 e 2, segundo BIBBLE; SINGHA (1993) e MUTSCHLER et al. (1992):

$C=\left(a^{2}+b^{2}\right)^{0,5}$

$H^{0}=\operatorname{arctg} \frac{b}{a}$

\subsection{Bases nitrogenadas voláteis}

Foi utilizada a técnica de BRASIL (1981). O resultado foi expresso em mg N.100g ${ }^{-1}$. 


\subsection{Capacidade de retenção de água (CRA)}

A capacidade de retenção de água (CRA) foi medida pela metodologia de centrifugação descrita por HOFFMANN; HAMM; BLUCHEL (1982) e a CRA calculada de acordo com a Equação 3:

$$
C R A=\frac{\left(P_{\text {após centrifugação }}-P_{\text {pós secagem }}\right)}{P_{\text {inicial }}} * 100
$$

\subsection{Análises estatísticas}

O delineamento estatístico utilizado foi inteiramente casualizado. Foi realizada a análise de variância pelo teste F, e a comparação das médias obtidas nos diferentes tratamentos foi analisada segundo teste de Tukey $(\mathrm{p}<0,01)$ (PIMENTELGOMES, 1982), com utilização do programa Statistical Analysis System (SAS INSTITUTE, 2003) através da ferramenta ANOVA. As análises foram realizadas em triplicata com três repetições.

\section{Resultados e discussão}

\subsection{Análise de cor}

As medidas de cor foram realizadas para se obter o efeito de diferentes doses de radiação, dietas, e tempo de armazenamento na carne de cordeiros (Tabela 1).

As medidas de cor para $a^{*}$ indicaram não haver diferenças estatísticas entre as doses de radiação utilizadas no experimento. Analisando outros tipos de carnes, Liu et al. (2003) e Nam; Ahn (2002), empregando doses de 0,2 a 5,0 kGy e 2,5 a 5,0 kGy, respectivamente, notaram aumento nos valores de $a^{*}$ quanto maior a dose de radiação empregada para estudos feitos com peito de frango e peru irradiados, diferindo dos resultados apresentados na Tabela 1. Para Faria (2005), que comparou diferentes raças de cordeiros, os valores de $a^{*}$ variaram de 9,86 a 10,33 , valores estes menores do que os encontrados na presente pesquisa. Quanto à armazenagem, os valores diminuíram ao longo do tempo, o que indica que as dietas empregadas não tiveram efeito antioxidante nas carnes (Tabela 1). Enquanto que para Du et al. (2000), a dieta com CLA (ácido linoleico conjugado) influenciou a coloração da carne de frango, provocando aumento nos valores de $a^{*}$.

Segundo Nam; Ahn (2002), a irradiação não afeta os valores de $b^{*}$ para peitos de peru embalados a vácuo, o que está de acordo com os resultados encontrados (Tabela 2), em que as diferentes doses de radiação não afetaram os valores de $b^{*}$. Somente aos 15 dias de estocagem, na dieta TAC1, percebeu-se que a dose de $2 \mathrm{kGy}$ apresentou valores maiores que para as demais doses o que pode ter sido provocado pela quantidade de taninos presente na dieta dos cordeiros.

Os valores foram afetados em relação ao tempo de armazenamento, somente após 15 dias de estocagem, o que está de acordo com Brewer (2004), no qual estudos com diferentes tipos de carnes, entre elas, carne de cordeiro, indicou que logo após o processo de irradiação não são observadas alterações, ocorrendo estas depois, no período de estocagem.
Tabela 1. Valores de $a^{*}$ medidos para a paleta (Infraspinatus, Triceps brachii, Supraspinatus) de cordeiros Santa Inês tratados com diferentes dietas e doses de radiação.

\begin{tabular}{|c|c|c|c|c|}
\hline Dieta & $\begin{array}{l}\text { Tratamento } \\
\text { (kGy) }\end{array}$ & $\begin{array}{c}\text { Tempo } 0 \\
(\text { dias })\end{array}$ & Tempo 15 (dias) & Prob $>$ F \\
\hline \multirow{3}{*}{ Controle } & 0 & $15,07 \pm 1,7^{\text {Ala2X3 }}$ & $7,60 \pm 3,5^{\mathrm{AbX}}$ & 0,01 \\
\hline & 2 & $15,40 \pm 1,7^{\mathrm{AaX}}$ & $5,97 \pm 2,6^{\mathrm{AbX}}$ & 0,01 \\
\hline & 4 & $16,47 \pm 4,5^{\mathrm{AaX}}$ & $6,30 \pm 2,6^{\mathrm{AbX}}$ & 0,01 \\
\hline \multirow{3}{*}{$\mathrm{TAC}^{*}$} & 0 & $14,90 \pm 1,7^{\mathrm{AaX}}$ & $9,17 \pm 2,6^{\mathrm{AaX}_{a}}$ & 0,01 \\
\hline & 2 & $16,07 \pm 3,6^{\mathrm{AaX}}$ & $5,40 \pm 4,5^{\mathrm{AbX}}$ & 0,01 \\
\hline & 4 & $14,53 \pm 2,6^{\mathrm{AaX}}$ & $4,13 \pm 3,3^{\mathrm{AbX}}$ & 0,01 \\
\hline \multirow{3}{*}{ TAC2* } & 0 & $15,67 \pm 2,6^{\mathrm{AaX}}$ & $9,40 \pm 3,6^{\mathrm{AaX}_{\mathrm{a}}}$ & 0,01 \\
\hline & 2 & $12,47 \pm 2,6^{\mathrm{AaX}}$ & $3,47 \pm 2,6^{\mathrm{AbX}}$ & 0,01 \\
\hline & 4 & $17,63 \pm 2,6^{\mathrm{AaX}_{\mathrm{a}}}$ & $9,27 \pm 2,6^{\mathrm{AaX}}$ & 0,01 \\
\hline \multirow{3}{*}{ Sorgo } & 0 & $10,37 \pm 2,6^{\mathrm{AaX}_{\mathrm{a}}}$ & $3,27 \pm 1,7^{\mathrm{AbX}}$ & 0,01 \\
\hline & 2 & $11,73 \pm 3,6^{\mathrm{AaX}}$ & $7,53 \pm 2,6^{\mathrm{AaX}}$ & 0,01 \\
\hline & 4 & $16,10 \pm 2,6^{\mathrm{AaX}}$ & $6,13 \pm 2,6^{\mathrm{AbX}}$ & 0,01 \\
\hline \multicolumn{5}{|c|}{$\begin{array}{l}\text { Letras maiúsculas diferentes nas colunas indicam que há diferença significativa entre } \\
\text { as doses. }\end{array}$} \\
\hline \multicolumn{5}{|c|}{$\begin{array}{l}{ }^{2} \text { Letras minúsculas diferentes (a-b) nas linhas indicam diferença significativa nos tempos } \\
\text { de armazenamento. }\end{array}$} \\
\hline \multicolumn{5}{|c|}{${ }^{3}$ Letras $\mathrm{X}, \mathrm{Y}$ indicam diferença estatística entre dietas. } \\
\hline
\end{tabular}

Tabela 2. Valores de $b^{*}$ medidos para a paleta (Infraspinatus, Triceps brachii, Supraspinatus) de cordeiros Santa Inês tratados com diferentes dietas e doses de radiação.

\begin{tabular}{|c|c|c|c|c|}
\hline Dieta & $\begin{array}{c}\text { Tratamento } \\
(\mathrm{kGy})\end{array}$ & $\begin{array}{c}\text { Tempo } 0 \\
\text { (dias) }\end{array}$ & $\begin{array}{l}\text { Tempo } 15 \\
\text { (dias) }\end{array}$ & Prob $>F$ \\
\hline \multirow{3}{*}{ Controle } & 0 & $7,80 \pm 1,7^{\mathrm{A} 1 \mathrm{a} 2 \mathrm{X} 3}$ & $12,70 \pm 3,5^{\mathrm{AbX}}$ & 0,01 \\
\hline & 2 & $6,93 \pm 1,7^{\mathrm{AaX}}$ & $11,03 \pm 2,6^{\mathrm{AbX}}$ & 0,01 \\
\hline & 4 & $6,30 \pm 4,5^{\mathrm{AaX}}$ & $13,87 \pm 2,6^{\mathrm{AbX}}$ & 0,01 \\
\hline \multirow{3}{*}{$\mathrm{TAC1}^{*}$} & 0 & $7,00 \pm 1,7^{\mathrm{AaX}}$ & $10,27 \pm 2,6^{\mathrm{AaX}}$ & 0,01 \\
\hline & 2 & $5,93 \pm 3,6^{\operatorname{AaX}}$ & $17,23 \pm 4,5^{\mathrm{BbX}}$ & 0,01 \\
\hline & 4 & $5,70 \pm 2,6^{\mathrm{AaX}}$ & $13,70 \pm 3,3^{\mathrm{ABbX}}$ & 0,01 \\
\hline \multirow{3}{*}{ TAC2* } & 0 & $6,20 \pm 2,6^{\mathrm{Aa}_{\mathrm{a}} \mathrm{X}}$ & $8,73 \pm 3,6^{\mathrm{A}_{\mathrm{a} X}}$ & 0,01 \\
\hline & 2 & $3,97 \pm 2,6^{\mathrm{AaX}}$ & $12,93 \pm 2,6^{\mathrm{AbX}}$ & 0,01 \\
\hline & 4 & $4,00 \pm 2,6^{\mathrm{AaX}}$ & $9,20 \pm 2,6^{\mathrm{AaX}}$ & 0,01 \\
\hline \multirow{3}{*}{ Sorgo } & 0 & $3,57 \pm 2,6^{\mathrm{AaX}}$ & $10,10 \pm 1,7^{\mathrm{AbX}}$ & 0,01 \\
\hline & 2 & $3,30 \pm 3,6^{\mathrm{AaX}}$ & $12,50 \pm 2,6^{\mathrm{AbX}}$ & 0,01 \\
\hline & 4 & $6,13 \pm 2,6^{\mathrm{AaX}}$ & $13,50 \pm 2,6^{\mathrm{AbX}}$ & 0,01 \\
\hline
\end{tabular}

${ }^{1}$ Letras maiúsculas diferentes nas colunas indicam que há diferença significativa entre as doses.

${ }^{2}$ Letras minúsculas diferentes (a-b) nas linhas indicam diferenças significativas nos tempos de armazenamento.

${ }^{3}$ Letras $\mathrm{X}, \mathrm{Y}$ indicam diferença estatística entre dietas.

${ }^{\star}$ Dietas suplementadas com 1,6 g. $\mathrm{Kg}^{-1}$ de tanino concentrado fornecidas por um dia (TAC1) e dois dias (TAC2). 
Segundo Liu et al. (2003), os valores de $b^{*}$ tendem a diminuir com o aumento das doses de radiação, o que se observa para o tempo inicial do experimento (Tempo 0). Porém, esta diminuição dos valores não representou diferença estatística entre as doses empregadas.

Valores de $b^{*}$ entre 6,83 e 8,15 foram encontrados por Faria (2005) trabalhando com diferentes grupos genéticos para carne de cordeiros, e estes valores estão entre os valores aqui encontrados.

Também não foram observadas diferenças estatísticas nos valores de $b^{*}$ quanto às dietas analisadas, o que concorda com os resultados encontrados por Du et al. (2000) ao estudar o efeito de dietas com CLA em frangos.

Os valores de $L^{*}$ não mostraram ser influenciados pelas doses de radiação aplicadas no experimento, porém, quanto ao tempo de armazenamento, demonstraram aumento significativo (Tabela 3), exceto para a dieta TAC2. Essa diferença pode ter ocorrido devido ao efeito antioxidante dos taninos condensados.

Segundo Faria (2005), os valores de $L^{*}$ estão entre 39,63 e 41,47 para cordeiros. Estes valores são menores do que os encontrados e podem ser explicados pelas dietas experimentais usadas serem diferentes e também pela diferença no peso de abate.

Esses resultados estão de acordo com Liu et al. (2003) e Nam; Ahn (2002), em que os valores de $L^{*}$ permaneceram estáveis nas diferentes doses de radiação estudadas.

As doses não afetaram significativamente os valores de $H$ da carne de cordeiro. Entretanto, o tempo de estocagem os influenciou significativamente (Tabela 4). Segundo Brewer (2004), esse comportamento é esperado, sendo mais significativo o tempo de armazenagem do que as doses para que ocorram alterações nos valores de $\mathrm{H}$.

De acordo com a Tabela 5, as doses estudadas não influenciaram significativamente os valores de C, porém o armazenamento mostrou ser significativo para todas as doses da dieta controle. A dieta TAC2 não apresentou alteração com o armazenamento, o que pode ter sido ocasionado pela influência da dieta na coloração da carne. TAC1 também apresentou maior estabilidade do $C$ com o armazenamento; havendo alteração com o armazenamento somente com a dose de $2 \mathrm{kGy}$.

Para todas as dietas analisadas os valores de $C$ não foram diferentes estatisticamente.

\subsection{Bases nitrogenadas voláteis}

Um método conveniente para avaliar a qualidade de carnes frescas é a determinação de bases voláteis.

O presente estudo demonstra que a irradiação, em ambos os tempos de armazenamento (tempo 0 e 15 dias), diminuiu significativamente os teores de bases voláteis das amostras, sendo a dose de $4 \mathrm{kGy}$ a que apresentou os menores valores (Tabela 6).

Quanto ao tempo de armazenamento os valores aumentaram, indiferentes às doses de radiação e ao tipo de dieta, com exceção para a dieta TAC2 nas doses de 2 e $4 \mathrm{kGy}$.
Tabela 3. Valores de $L^{*}$ medidos para a paleta (Infraspinatus, Triceps brachii, Supraspinatus) de cordeiros Santa Inês tratados com diferentes dietas e doses de radiação.

\begin{tabular}{lcccc}
\hline Dieta & $\begin{array}{c}\text { Tratamento } \\
(\mathrm{kGy})\end{array}$ & $\begin{array}{c}\text { Tempo } 0 \\
(\text { dias })\end{array}$ & $\begin{array}{c}\text { Tempo } 15 \\
\text { (dias) }\end{array}$ & Prob $>$ F \\
\hline \multirow{4}{*}{ Controle } & 0 & $50,97 \pm 1,7^{\mathrm{Ala} a X 3}$ & $59,17 \pm 3,5^{\mathrm{AbX}}$ & 0,01 \\
& 2 & $51,97 \pm 1,7^{\mathrm{AaX}}$ & $55,93 \pm 2,6^{\mathrm{AbX}}$ & 0,01 \\
& 4 & $47,10 \pm 4,5^{\mathrm{AaX}}$ & $54,67 \pm 2,6^{\mathrm{AbX}}$ & 0,01 \\
& 0 & $50,33 \pm 1,7^{\mathrm{AaXY}}$ & $56,20 \pm 2,6^{\mathrm{AaX}}$ & 0,01 \\
TAC1 $^{*}$ & 2 & $57,00 \pm 3,6^{\mathrm{AaX}}$ & $61,70 \pm 4,5^{\mathrm{AbXY}}$ & 0,01 \\
& 4 & $48,17 \pm 2,6^{\mathrm{AaX}}$ & $56,20 \pm 3,3^{\mathrm{AaX}}$ & 0,01 \\
& & & & \\
& 0 & $45,77 \pm 2,6^{\mathrm{AaY}}$ & $54,17 \pm 3,6^{\mathrm{AaX}}$ & 0,01 \\
TAC2 $^{*}$ & 2 & $49,70 \pm 2,6^{\mathrm{AaX}}$ & $71,20 \pm 2,6^{\mathrm{BaY}}$ & 0,01 \\
& 4 & $47,37 \pm 2,6^{\mathrm{AaX}}$ & $55,00 \pm 2,6^{\mathrm{AaX}}$ & 0,01 \\
& & & & \\
& 0 & $51,60 \pm 2,6^{\mathrm{AaX}}$ & $65,67 \pm 1,7^{\mathrm{AbX}}$ & 0,01 \\
Sorgo & 2 & $50,17 \pm 3,6^{\mathrm{AaX}}$ & $57,47 \pm 2,6^{\mathrm{AaX}}$ & 0,01 \\
& 4 & $46,43 \pm 2,6^{\mathrm{AaX}}$ & $57,77 \pm 2,6^{\mathrm{AbX}}$ & 0,01 \\
\hline
\end{tabular}

${ }^{1}$ Letras maiúsculas diferentes nas colunas indicam que há diferença significativa entre as doses.

${ }^{2}$ Letras minúsculas diferentes (a-b) nas linhas indicam diferenças significativas nos tempos de armazenamento.

${ }^{3}$ Letras $\mathrm{X}, \mathrm{Y}$ indicam diferença estatística entre dietas.

${ }^{\star}$ Dietas suplementadas com 1,6 g. $\mathrm{Kg}^{-1}$ de tanino concentrado fornecidas por um dia (TAC1) e dois dias (TAC2).

Tabela 4. Valores de $H$ medidos para a paleta (Infraspinatus, Triceps brachii, Supraspinatus) de cordeiros Santa Inês tratados com diferentes dietas e doses de radiação.

\begin{tabular}{lcccc}
\hline Dieta & $\begin{array}{c}\text { Tratamento } \\
(\mathrm{kGy})\end{array}$ & $\begin{array}{c}\text { Tempo } 0 \\
(\text { dias })\end{array}$ & $\begin{array}{c}\text { Tempo } 15 \\
\text { (dias) }\end{array}$ & Prob $>$ F \\
\hline \multirow{4}{*}{ Controle } & 0 & $27,80 \pm 1,7^{\mathrm{Ala2X} 3}$ & $59,24 \pm 3,5^{\mathrm{AbX}}$ & 0,01 \\
& 2 & $24,67 \pm 1,7^{\mathrm{AaX}}$ & $61,64 \pm 2,6^{\mathrm{AbX}}$ & 0,01 \\
& 4 & $20,88 \pm 4,5^{\mathrm{AaX}}$ & $65,72 \pm 2,6^{\mathrm{AbX}}$ & 0,01 \\
& & & & \\
TAC1 $^{*}$ & 2 & $25,43 \pm 1,7^{\mathrm{AaX}}$ & $48,61 \pm 2,6^{\mathrm{AbX}}$ & 0,01 \\
& 4 & $21,22 \pm 3,6^{\mathrm{AaX}}$ & $72,51 \pm 4,5^{\mathrm{AbX}}$ & 0,01 \\
& & & & 0,01 \\
& 0 & $21,68 \pm 2,6^{\mathrm{AaX}}$ & $46,55 \pm 3,6^{\mathrm{AbX}}$ & 0,01 \\
TAC2 $^{*}$ & 2 & $18,35 \pm 2,6^{\mathrm{AaX}}$ & $76,12 \pm 2,6^{\mathrm{AbX}}$ & 0,01 \\
& 4 & $11,41 \pm 2,6^{\mathrm{AaX}}$ & $44,93 \pm 2,6^{\mathrm{AbY}}$ & 0,01 \\
& & & & \\
& 0 & $18,89 \pm 2,6^{\mathrm{AaX}}$ & $73,67 \pm 1,7^{\mathrm{AbX}}$ & 0,01 \\
Sorgo & 2 & $13,87 \pm 3,6^{\mathrm{AaX}}$ & $59,02 \pm 2,6^{\mathrm{AbX}}$ & 0,01 \\
& 4 & $22,07 \pm 2,6^{\mathrm{AaX}}$ & $65,10 \pm 2,6^{\mathrm{AbXY}}$ & 0,01 \\
\hline
\end{tabular}

${ }^{1}$ Letras maiúsculas diferentes nas colunas indicam que há diferença significativa entre as doses.

${ }^{2}$ Letras minúsculas diferentes (a-b) nas linhas indicam diferenças significativas nos tempos de armazenamento.

${ }^{3}$ Letras $\mathrm{X}, \mathrm{Y}$ indicam diferença estatística entre dietas.

${ }^{\star}$ Dietas suplementadas com 1,6 g. $\mathrm{Kg}^{-1}$ de tanino concentrado fornecidas por um dia (TAC1) e dois dias (TAC2). 
Estes resultados estão de acordo com Al-Bachir, Mehio (2001) que, estudando hambúrguer irradiado e não irradiado com diferentes tempos de armazenamento, encontraram o mesmo comportamento.

Os valores encontrados indicaram que as amostras estavam em estado de deterioração, sendo impróprias para consumo, conforme Pearson (1976), que determina que valores abaixo de $20 \mathrm{mg} \mathrm{N} \cdot 100 \mathrm{~g}^{-1}$ de amostra é indicativo de carne fresca, e acima ou

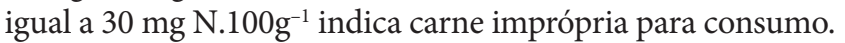

Quanto às dietas estudadas nas doses 0 e $2 \mathrm{kGy}$, a que apresentou os menores valores foram as dietas com sorgo, no tempo inicial de armazenamento. Para a dose de $4 \mathrm{kGy}$, os valores de bases nitrogenadas voláteis se mostraram indiferentes às dietas estudadas (Figura 1).

Na Figura 2, observa-se que, aos 15 dias de estocagem, a dieta controle foi a que mais sofreu influência nas doses de 0 e $4 \mathrm{kGy}$, sendo os teores de bases voláteis maiores 55,47 e $47,46 \mathrm{mg} \mathrm{N} .100 \mathrm{~g}^{-1}$ de paleta de cordeiro. Para a dose de $2 \mathrm{kGy}$, tanto a dieta controle quanto a dieta TAC1 apresentaram os maiores valores, bem se diferenciaram estatisticamente das demais dietas.

\subsection{CRA}

Dependendo da dose, várias mudanças organolépticas podem alterar a carne irradiada. A textura e a capacidade de retenção de água são alteradas pela desnaturação das proteínas estruturais (LAWRIE, 1985). As doses empregadas diminuíram os valores de CRA, no início do armazenamento. Após o armazenamento (15 dias), o comportamento com a irradiação se alterou devido a outras alterações que ocorrem no tecido muscular (Tabela 7). O armazenamento também influenciou nesses teores, exceto para a dieta TAC1. O pH e a organização estrutural das proteínas do músculo são decisivos

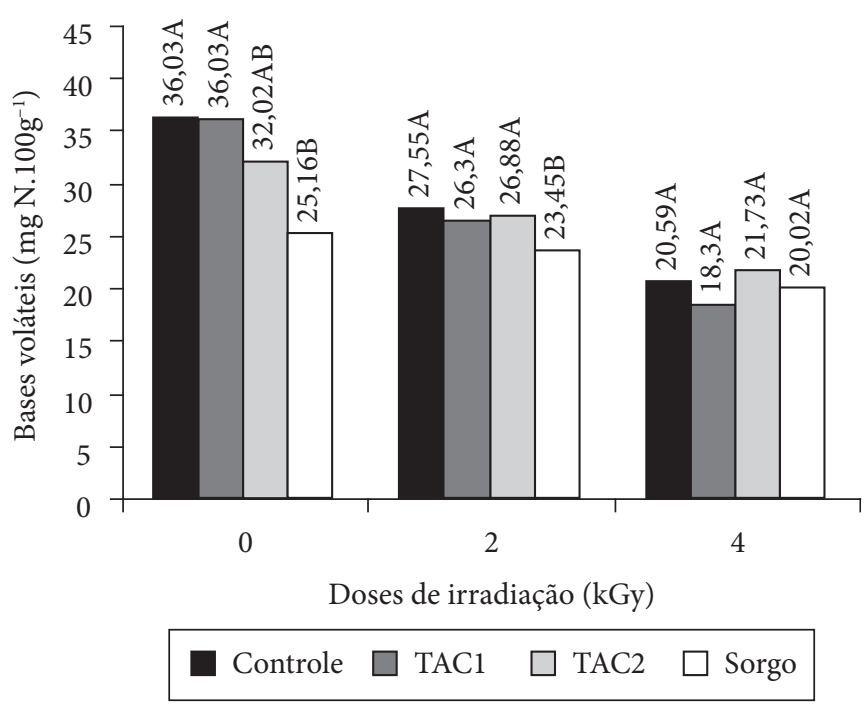

Figura 1. Efeito das diferentes dietas estudadas nos teores de bases voláteis no tempo inicial de armazenamento (0 dias).
Tabela 5. Valores de $C$ medidos para a paleta (Infraspinatus, Triceps brachii, Supraspinatus) de cordeiros Santa Inês tratados com diferentes dietas e doses de radiação.

\begin{tabular}{|c|c|c|c|c|}
\hline Dieta & $\begin{array}{c}\text { Tratamento } \\
(\mathrm{kGy})\end{array}$ & $\begin{array}{c}\text { Tempo } 0 \\
\text { (dias) }\end{array}$ & $\begin{array}{l}\text { Tempo } 15 \\
\text { (dias) }\end{array}$ & Prob $>F$ \\
\hline \multirow{3}{*}{ Controle } & 0 & $17,02 \pm 1,7^{\text {Ala2X3 }}$ & $14,81 \pm 3,5^{\mathrm{AbX}}$ & 0,01 \\
\hline & 2 & $16,95 \pm 1,7^{\mathrm{AaX}}$ & $12,65 \pm 2,6^{\mathrm{AbX}}$ & 0,01 \\
\hline & 4 & $17,66 \pm 4,5^{\mathrm{AaX}}$ & $15,24 \pm 2,6^{\mathrm{AbX}}$ & 0,01 \\
\hline \multirow{3}{*}{$\mathrm{TAC}^{*}$} & 0 & $16,47 \pm 1,7^{\mathrm{AaX}}$ & $13,96 \pm 2,6^{\mathrm{AaX}}$ & 0,01 \\
\hline & 2 & $17,23 \pm 3,6^{\mathrm{AaX}}$ & $18,28 \pm 4,5^{\mathrm{AbX}}$ & 0,01 \\
\hline & 4 & $15,91 \pm 2,6^{\mathrm{A}_{\mathrm{a} X}}$ & $14,45 \pm 3,3^{A_{a} X}$ & 0,01 \\
\hline \multirow{3}{*}{$\mathrm{TAC}^{*}$} & 0 & $16,88 \pm 2,6^{\mathrm{AaX}_{\mathrm{a}}}$ & $13,30 \pm 3,6^{\mathrm{AaX}_{\mathrm{a}}}$ & 0,01 \\
\hline & 2 & $13,09 \pm 2,6^{\mathrm{AaX}}$ & $13,51 \pm 2,6^{\mathrm{AaX}}$ & 0,01 \\
\hline & 4 & $18,22 \pm 2,6^{\mathrm{AaX}_{\mathrm{a}}}$ & $13,07 \pm 2,6^{\mathrm{AaX}}$ & 0,01 \\
\hline \multirow{3}{*}{ Sorgo } & 0 & $11,07 \pm 2,6^{\mathrm{A}_{\mathrm{a}} \mathrm{X}}$ & $10,79 \pm 1,7^{\mathrm{AbX}}$ & 0,01 \\
\hline & 2 & $12,51 \pm 3,6^{\mathrm{AaX}}$ & $14,66 \pm 2,6^{\mathrm{AaX}}$ & 0,01 \\
\hline & 4 & $17,38 \pm 2,6^{\operatorname{AaX}}$ & $15,00 \pm 2,6^{\mathrm{AbX}}$ & 0,01 \\
\hline
\end{tabular}

${ }^{1}$ Letras maiúsculas diferentes nas colunas indicam que há diferença significativa entre as doses.

${ }^{2}$ Letras minúsculas diferentes (a-b) nas linhas indicam diferenças significativas nos tempos de armazenamento.

${ }^{3}$ Letras $\mathrm{X}, \mathrm{Y}$ indicam diferença estatística entre dietas.

${ }^{\star}$ Dietas suplementadas com 1,6 g. $\mathrm{Kg}^{-1}$ de tanino concentrado fornecidas por um dia (TAC1) e dois dias (TAC2).

Tabela 6. Efeito de diferentes doses de radiação, dietas e tempo de armazenamento nos valores de bases nitrogenadas voláteis encontradas para paleta (Infraspinatus, Triceps brachii, Supraspinatus) de cordeiro Santa Inês.

\begin{tabular}{ccccc}
\hline Dieta & $\begin{array}{c}\text { Tratamento } \\
(\mathrm{kGy})\end{array}$ & $\begin{array}{c}\text { Tempo } 0 \\
\text { (dias) }\end{array}$ & $\begin{array}{c}\text { Tempo } 15 \\
\text { (dias) }\end{array}$ & Prob $>$ F \\
\hline \multirow{3}{*}{ Controle } & 0 & $36,03 \pm 1,7^{\mathrm{Ala} 2}$ & $55,47 \pm 3,5^{\mathrm{Ab}}$ & 0,01 \\
& 2 & $27,55 \pm 1,7^{\mathrm{ABa}}$ & $42,32 \pm 2,6^{\mathrm{Bb}}$ & 0,01 \\
& 4 & $20,59 \pm 4,5^{\mathrm{Ba}}$ & $47,46 \pm 2,6^{\mathrm{ABb}}$ & 0,01 \\
& & & & \\
TAC1 $^{*}$ & 0 & $36,03 \pm 1,7^{\mathrm{Aa}}$ & $40,60 \pm 2,6^{\mathrm{Aa}}$ & 0,01 \\
& 2 & $26,30 \pm 3,6^{\mathrm{ABa}}$ & $42,89 \pm 4,5^{\mathrm{ABb}}$ & 0,01 \\
& 4 & $18,30 \pm 2,6^{\mathrm{Ba}}$ & $27,45 \pm 3,3^{\mathrm{Ba}}$ & 0,01 \\
& & & & \\
TAC2 $^{*}$ & 0 & $32,02 \pm 2,6^{\mathrm{Aa}}$ & $41,74 \pm 3,6^{\mathrm{Aa}}$ & 0,01 \\
& 2 & $26,88 \pm 2,6^{\mathrm{ABa}}$ & $26,30 \pm 2,6^{\mathrm{Ba}}$ & 0,01 \\
& 4 & $21,73 \pm 2,6^{\mathrm{Ba}}$ & $24,59 \pm 2,6^{\mathrm{Ba}}$ & 0,01 \\
& & & & \\
Sorgo & 0 & $25,16 \pm 2,6^{\mathrm{Aa}}$ & $36,02 \pm 1,7^{\mathrm{Ab}}$ & 0,01 \\
& 2 & $23,45 \pm 3,6^{\mathrm{Aa}}$ & $28,02 \pm 2,6^{\mathrm{Aa}}$ & 0,01 \\
& 4 & $20,02 \pm 2,6^{\mathrm{Aa}}$ & $30,31 \pm 2,6^{\mathrm{Ab}}$ & 0,01 \\
\hline
\end{tabular}

${ }^{1}$ Letras maiúsculas diferentes nas colunas indicam que há diferença significativa entre as doses.

${ }^{2}$ Letras minúsculas diferentes (a-b) nas linhas indicam diferenças significativas nos tempos de armazenamento.

${ }^{\star}$ Dietas suplementadas com 1,6 g. $\mathrm{Kg}^{-1}$ de tanino concentrado fornecidas por um dia (TAC1) e dois dias (TAC2). 
para a distribuição da água na carne, o que afeta diretamente o CRA (FIK; SURÓWKA; FIREK, 2008; HUFF-LONERGAN; LONERGAN, 2005; KRISTENSEN; PURSLOW, 2001; MELODY et al., 2004).

As diferentes dietas influenciaram bastante o CRA da paleta. Sendo as dietas TAC1 e TAC2 as que mais perderam água

Tabela 7. Efeito de diferentes doses de radiação e tempo de armazenamento na capacidade de retenção de água (CRA) de paletas (Infraspinatus, Triceps brachii, Supraspinatus) de cordeiros Santa Inês.

\begin{tabular}{ccccc}
\hline Dieta & $\begin{array}{c}\text { Tratamento } \\
(\mathrm{kGy})\end{array}$ & $\begin{array}{c}\text { Tempo 0 } \\
(\text { dias })\end{array}$ & $\begin{array}{c}\text { Tempo } 15 \\
(\text { dias })\end{array}$ & Prob $>$ F \\
\hline \multirow{3}{*}{ Controle } & 0 & $54,83 \pm 1,00^{\mathrm{Ala} 2}$ & $57,33 \pm 1,53^{\mathrm{Aa}}$ & 0,01 \\
& 2 & $58,64 \pm 0,60^{\mathrm{Ba}}$ & $47,33 \pm 0,58^{\mathrm{Bb}}$ & 0,01 \\
& 4 & $51,65 \pm 0,60^{\mathrm{Ca}}$ & $51,00 \pm 1,00^{\mathrm{Ba}}$ & 0,01 \\
& & & & \\
TAC1 $^{*}$ & 2 & $58,70 \pm 0,60^{\mathrm{Ba}}$ & $59,00 \pm 1,73^{\mathrm{Aa}}$ & 0,01 \\
& 4 & $56,02 \pm 0,90^{\mathrm{Ca}}$ & $59,00 \pm 2,65^{\mathrm{Aa}}$ & 0,01 \\
& & & & \\
& 0 & $61,13 \pm 1,00^{\mathrm{Aa}}$ & $65,00 \pm 2,00^{\mathrm{Aa}}$ & 0,01 \\
TAC2 $^{*}$ & 2 & $45,75 \pm 0,22^{\mathrm{Ba}}$ & $49,00 \pm 2,65^{\mathrm{Ba}}$ & 0,01 \\
& 4 & $51,44 \pm 0,51^{\mathrm{Ca}}$ & $54,33 \pm 0,58^{\mathrm{Bb}}$ & 0,01 \\
& & & & \\
Sorgo & 0 & $50,55 \pm 0,58^{\mathrm{Aa}}$ & $61,67 \pm 2,52^{\mathrm{Ab}}$ & 0,01 \\
& 2 & $51,05 \pm 0,04^{\mathrm{Aa}}$ & $61,67 \pm 1,53^{\mathrm{Ab}}$ & 0,01 \\
& 4 & $49,48 \pm 0,14^{\mathrm{Aa}}$ & $43,33 \pm 0,58^{\mathrm{Bb}}$ & 0,01 \\
\hline
\end{tabular}

${ }^{1}$ Letras maiúsculas diferentes nas colunas indicam que há diferença significativa entre as doses.

${ }^{2}$ Letras minúsculas diferentes (a-b) nas linhas indicam diferenças significativas nos tempos de armazenamento.

${ }^{*}$ Dietas suplementadas com $1,6 \mathrm{~g} \cdot \mathrm{Kg}^{-1}$ de tanino altamente concentrado fornecidas por um dia (TAC1) e dois dias (TAC2).

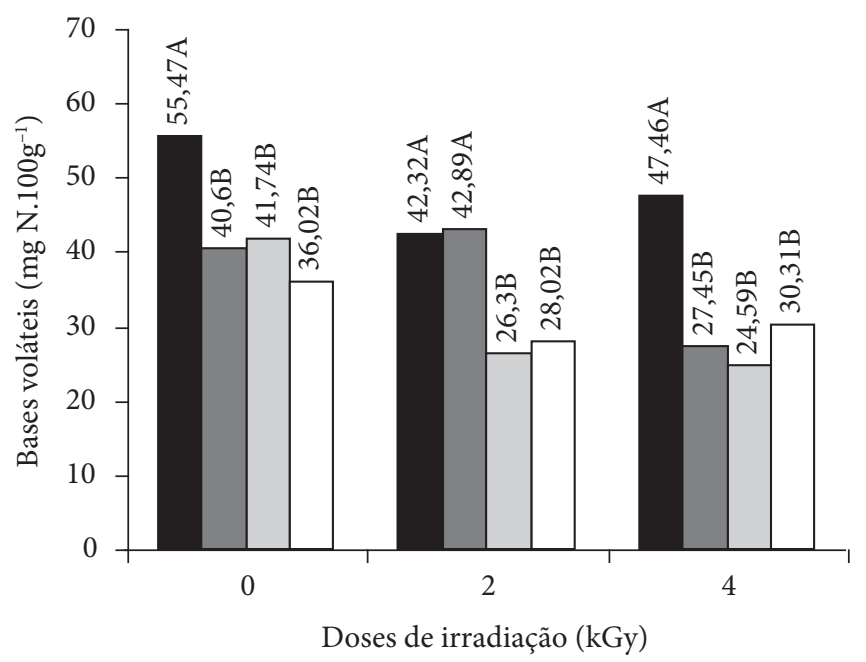

Controle $\square$ TAC1 $\square$ TAC2 $\square$ Sorgo

Figura 2. Efeito das diferentes dietas estudadas nos teores de bases voláteis no tempo final de armazenamento (15 dias). para a dose 0 kGy (Figura 3). Para a dose de $2 \mathrm{kGy}$, as dietas TAC2 e sorgo foram as que menos perderam, se diferenciando estatisticamente das demais dietas. Na dose de $4 \mathrm{kGy}$, a dieta TAC1 foi a mais afetada, para o tempo inicial de armazenamento (0 dias). O que indica que, entre todas as dietas estudadas, a TAC1 foi a que apresentou a menor capacidade de retenção de água.

Na Figura 4, é observada a capacidade de retenção de água para o tempo final de armazenamento $(\mathrm{T}=15$ dias), na qual a dieta que apresentou os maiores teores de CRA foi a TAC1.

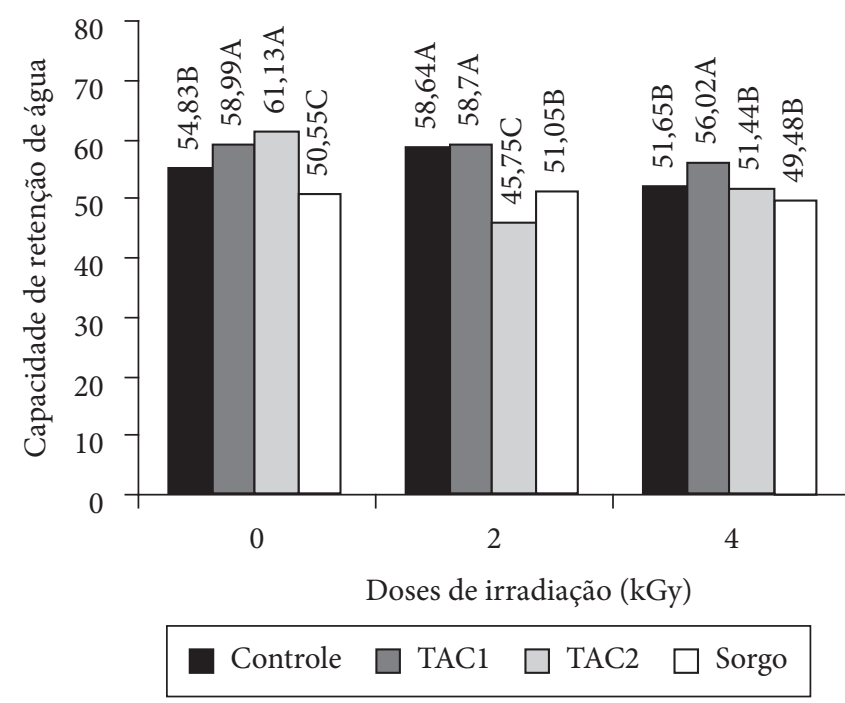

Figura 3. Efeito de diferentes dietas na capacidade de retenção de água no tempo inicial de armazenamento (0 dias).

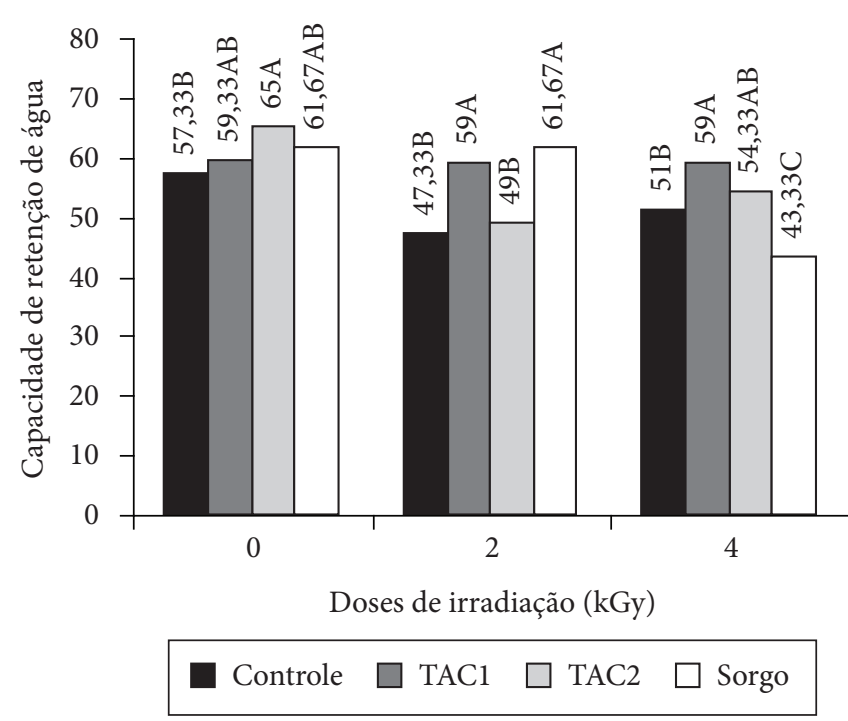

Figura 4. Efeito das diferentes dietas na capacidade de retenção de água no tempo final de armazenamento (15 dias). 


\section{Conclusão}

A irradiação é um bom método para ser aplicado a carnes, visto que a qualidade do produto pode ser mantida durante a estocagem, embora tenham ocorrido alterações nas bases nitrogenadas e CRA. A dieta fornecida aos animais influenciou nos resultados de $\mathrm{H}$, bases voláteis e CRA, provocando mudanças na qualidade. Sempre que possível a informação da composição da dieta consumida deverá ser levada em consideração, para um melhor entendimento das mudanças químicas que ocorrem nas carnes.

\section{Referências bibliográficas}

AL-BACHIR, M.; MEHIO, A. Irradiated luncheon meat: microbiological, chemical and sensory characteristics during storage. Food Chemistry, v. 75, n. 2, p. 169-175, 2001.

ANDERSON, J. B. Minerais. In: MAHAN, L. K.; ESCOTT-STUMP, S.; KRAUSE, M. V. Alimentos, Nutrição e Dietoterapia. São Paulo: Roca, 2005.

BIBBLE, B. B.; SINGHA, S. Canopy position influences CIELAB coordinates of peach color. HortScience, v. 28, n. 10, p. 992-993, 1993.

BRASIL. Ministério da Agricultura. Secretaria Nacional de Defesa Agropecuária. Laboratório Nacional de Referência Animal. Métodos analíticos para oficias de controle de produtos de origem animal e seus ingredientes: II - Métodos físicos e químicos. Brasília, 1981.

BREWER, S. Irradiation effects on meat color - a review. Meat Science, v. 68, n. 1, p. 1-17, 2004.

BRUNE, M.; ROSSANDER, L.; HALLBERG, L. Iron absorption and phenolic compounds: importance of different phenolic structures. Journal of Clinical Nutrition, v. 43, n. 8, p. 547-558, 1989.

DU, M. et al. Effect of dietary sorghum cultivars on the storage stability of broiler breast and thigh meat. Poultry Science, v. 81, n. 9, p. 1385-1391, 2002.

DU, M. et al. Influence of dietary conjugated linoleic acid on volatile profiles, color and lipid oxidation of irradiated raw chicken meat. Meat Science, v. 56, n. 4, p. 387-395, 2000.

FARIA, P. B. Efeito de diferentes grupos genéticos sobre parâmetros quantitativos e qualitativos da carne de cordeiros. Lavras, 2005. 62 p. Dissertação (Mestrado em Ciência dos Alimentos) Universidade Federal de Lavras - UFLA.

FIK, M.; SURÓWKA, K.; FIREK, B. Properties of refrigerated ground beef treated with potassium lactate and sodium diacetate. Journal of the Science of Food and Agriculture, v. 88, n. 1, p. 91-99, 2008.

HOFFMANN, H.; HAMM, R.; BLUCHEL, E. Neus uber die bestimung der wasserbinding des nut hielf filter paper premethods. Fleishwirtchaft, v. 62, n. 1, p. 87-94, 1982.
HUFF-LONERGAN, E.; LONERGAN, S. M. Mechanisms of waterholding capacity of meat: the role of post mortem biochemical and structural changes. Meat Science, v. 71, n. 1, p. 194-204, 2005.

LAWRIE, R. A. The eating quality of meat. In: Meat Science. 5 ed. New York: Pergamon Press, 2002

LAWRIE, R. A. Meat science. Oxford [Oxfordshire]; New York: Pergamon Press, 1985. 267 p.

LIU, Y. et al. Changes in structure and color characteristics of irradiated chicken breast as a function of dosage and storage time. Meat Science, v. 63, n. 3, p. 301-330, 2003.

KRISTENSEN, L.; PURSLOW, P. P. The effect of ageing on the waterholding capacity of pork: role of cytoskeletal proteins. Meat Science, v. 58, n. 1, p. $17-23,2001$.

MELODY, J. L. et al. Early post mortem biochemical factors influence tenderness and water-holding capacity of three porcine muscles. Journal of Animal Science, v. 82, n. 4, p. 1195-1205, 2004.

MUTSCHLER, M. A. et al. Toamyo fruit quality and shelf life in hybrids heterozigous for the alc ripening mutant. HortScience, v. 27, n. 4, p. $352-355,1992$.

NAM, K. C.; AHN, D. U. Carbon monoxide-heme pigment is responsible for the pink color in irradiated raw turkey breast meat. Meat Science, v. 60, n. 1, p. 25-33, 2002.

NAM, K. C.; PRUSA, K. J.; AHN, D. U. Addition of antioxidant to improve a quality and sensory characteristics of irradiated pork patties. In: ANNUAL MEETING OF THE INSTITUTE OF FOOD TECHNOLOGISTS, Anaheim, CA, 2002. Proceedings... (Paper 88-89).

NAM, K. C. et al. Effect of dietary vitamin $\mathrm{E}$ and irradiation on lipid oxidation, color and volatiles fresh and previously frozen turkey breast patties. Meat Science, v. 65, n. 1, p. 513-521, 2003.

PEARSON, D. Técnicas de laboratorio para el análisis de alimentos. Zaragoza: Acribia, 1976. $331 \mathrm{p}$.

PIMENTEL-GOMES, F. Curso de estatística experimental. 10 ed. São Paulo: Nobel, 1982.

SAS Institute. SAS user's guide: statistic. Version 6. 12 ed. Cary, 2003. $846 \mathrm{p}$.

SILVA, M. L. Efeito de dois métodos de cocção - água e vapor - nos parâmetros de qualidade do músculo semitendinosus. Piracicaba, 2004. 102 p. Dissertação (Mestrado em Agronomia) - Escola Superior de Agricultura "Luiz de Queiroz" - ESALQ.

SOARES, S. E. Ácidos fenólicos como antioxidantes. Revista de Nutrição, v. 15, n. 1, p. 71-81, 2002.

XIONG, Y. L. et al. Gelation of crude myofibrillar proteins isolated from beef heart under antioxidant conditions. Journal of Food Science, v. 58, n. 6, p. 1241-1244, 1993. 